\title{
The Hydrodynamic Limit of Nonlinear Fokker-Planck Equation
}

\author{
Yi’ang Ren, Lijuan Yu, Jie Liao* \\ Department of Mathematics, East China University of Science and Technology, Shanghai, China \\ Email: *liaojie@ecust.edu.cn
}

How to cite this paper: Ren, Y.A., Yu, L.J. and Liao, J. (2020) The Hydrodynamic Limit of Nonlinear Fokker-Planck Equation. Journal of Applied Mathematics and Physics, 8, 2488-2499. https://doi.org/10.4236/jamp.2020.811184

Received: October 24, 2020

Accepted: November 23, 2020

Published: November 26, 2020

Copyright $\odot 2020$ by author(s) and Scientific Research Publishing Inc. This work is licensed under the Creative Commons Attribution International License (CC BY 4.0).

http://creativecommons.org/licenses/by/4.0/

\section{(c) (i) Open Access}

\begin{abstract}
The non-linear Fokker-Planck equation arises in describing the evolution of stochastic system, which is a variant of the Boltzmann equation modeling the evolution of the random system with Brownian motion, where the collision term is replaced by a drift-diffusion operator. This model conserves mass, momentum and energy; the dissipation is much weaker than that in a simplified model we considered before which conserved only mass, thus more difficult to analyze. The macro-micro decomposition of the solution around the local Maxwellian introduced by T.-P. Liu, T. Yang and S.-H. Yu for Boltzmann equation is used, to reformulate the model into a fluid-type system incorporate viscosity and heat diffusion terms, coupled with an equation of the microscopic part. The viscosity and heat diffusion terms can give dissipative mechanism for the analysis of the model.
\end{abstract}

\section{Keywords}

Non-Linear Fokker-Planck Equation, Macro-Micro Decomposition, Fluid-Type System, Viscosity, Heat Diffusion

\section{Introduction}

The non-linear Fokker-Planck equations arise in describing the evolution of stochastic system such as the erratic motions of small particles immersed in fluids, fluctuations of the intensity of laser light, velocity distributions of fluid particles in turbulent flows, or the stochastic behavior of exchange rates. This model has been widely applied in physics, biology, ecology, economy and social science, see [1]-[6] and references therein. In this paper, we study the fully non-linear Fokker-Planck equation [7]

$$
\partial_{t} f+v \cdot \nabla_{x} f=\rho^{\alpha} \nabla_{v} \cdot\left[T \nabla_{v} f+(v-u) f\right],
$$


where the unknown function $f=f(t, x, v)$ is the distribution of particles at time $t$, position $x$ and velocity $v$ for $(t, x, v) \in \mathbb{R}_{+} \times \mathbb{R}^{3} \times \mathbb{R}^{3}, \alpha \in[0,1]$ is the friction parameter, $\rho, \bar{u}, T$ are the mass density, the mean velocity and the local temperature, which are coupled to $f$ by

$$
\begin{aligned}
& \rho(t, x)=\int_{\mathbb{R}^{3}} f(t, x, v) \mathrm{d} v, \rho u(t, x)=\int_{\mathbb{R}^{3}} v f(t, x, v) \mathrm{d} v, \\
& 3 \rho T(t, x)=\int_{\mathbb{R}^{3}}|v-u|^{2} f(t, x, v) \mathrm{d} v .
\end{aligned}
$$

As a variant of the Boltzmann equation modeling the evolution of the random system with Brownian motion, the collision term is replaced by a drift-diffusion operator. Note that this famous model conserves mass, momentum and energy [8] [9], and the right hand side of the equation is strongly non-linear, more precisely, the dissipation is much weaker than that in a simplified model considered in [10], thus more difficult to analyze.

Some related models. When relativistic/quantum effect is included, the relativistic/quantum Fokker-Planck equations are considered in [11] [12] [13] [14] and references therein, and the famous Fokker-Planck-Boltzmann considered both Boltzmann collision operator and Fokker-Planck operator, see [15] [16] [17] and references therein. We mention also that the Fokker-Planck equation can be coupled with other external field, thus Vlasov-type Fokker-Planck equations with an additional force term are considered. For example, the Vlasov-Poisson-Fokker-Planck model incorporates the self-consistent effects described by mean-field interactions, when the interaction is Coulomb [18] [19]. Moreover, when the Vlasov-Fokker-Planck equation is coupled with Maxwell equations, Euler equations or Navier-Stokes equations, respectively, we have Vlasov-Maxwell-Fokker-Planck model (see [20] and references therein), Vlasov-Euler-Fokker-Planck system (see [21] and references therein), and Vlasov-Fokker-Planck-Navier-Stokes system (see [22] [23] [24] and references therein).

Motivation of the present study. Note that this fully non-linear model (1.1) preserves mass, momentum and energy; the dissipation is much weaker than that in the simplified model considered in [25], thus more difficult to analyze. The aim of the present study is to recover the dissipative nature of the model, and establish the connection between the fully non-linear Fokker-Planck equation and the Boltzmann equation. That is, we use the macro-micro decomposition of the solution around the local Maxwellian introduced in [25] for Boltzmann equation, to reformulate the model into a fluid-type system coupled with an equation of the microscopic part. Especially, by careful computation, we derived the viscosity and heat diffusion terms in the fluid-type system from the microscopic part, which give the dissipative mechanism for the analysis of the model.

\section{Macro-Micro Decomposition of the Solution}

Following [25], we decompose the unknown function $f$ around the local Max- 
wellian as

$$
f(t, x, v)=M(t, x, v)+G(t, x, v),
$$

where the local Maxwellian $M=M(t, x, v)=M_{[\rho, u, T]}(v)$ depending on the macroscopic quantities $(\rho, u, T)$ defined in (1.2), is given by

$$
M_{[\rho, u, T]}(v)=\frac{\rho}{(2 \pi T)^{3 / 2}} \exp \left(-\frac{|v-u|^{2}}{2 T}\right),
$$

and $G(t, x, v)$ is the microscopic, non-fluid part. Define the weighted inner product

$$
\langle g, h\rangle_{M}:=\int_{\mathbb{R}^{3}} g(v) h(v) \frac{1}{M} \mathrm{~d} v
$$

for two functions $g$ and $h$ of $v$ such that the integral is well defined. Denote

$$
\chi_{0}^{M}=\frac{1}{\sqrt{\rho}} M, \quad \chi_{i}^{M}=\frac{v_{i}-u_{i}}{\sqrt{T \rho}} M(i=1,2,3), \quad \chi_{4}^{M}=\frac{1}{\sqrt{6 \rho}}\left(\frac{|v-u|^{2}}{T}-3\right) M,
$$

which are orthonormal with respect to the inner product (2.3), that is,

$$
\left\langle\chi_{\beta}^{M}, \chi_{\gamma}^{M}\right\rangle_{M}=\delta_{\beta \gamma}, \beta, \gamma=0,1,2,3,4 .
$$

Then the macroscopic projection $P_{0}^{M}$ and microscopic projection $P_{1}^{M}$ of a function $h$ are defined as [25]:

$$
P_{0}^{M} h:=\sum_{\beta=0}^{4}\left\langle h, \chi_{\beta}^{M}\right\rangle_{M} \chi_{\beta}^{M}, P_{1}^{M} h=\left(I-P_{0}^{M}\right) h .
$$

The projections will be used to decompose the nonlinear Fokker-Planck Equation (1.1) into a system of fluid equations coupled with a microscopic equation.

\section{Decomposition of the Equation}

Take the right hand side of (1.1) as the "collision term", it has five collision invariants $\left\{\psi_{\beta}\right\}_{\beta=0}^{4}$, where

$$
\psi_{0}=1, \psi_{i}=v_{i}(i=1,2,3), \psi_{4}=\frac{1}{2}|v|^{2},
$$

thus, by multiplying Equation (1.1) with collision invariants and integrating, one has five conservation laws

$$
\int_{\mathbb{R}^{3}} \psi_{\beta}\left(f_{t}+v \cdot \nabla_{x} f\right) \mathrm{d} v=0, \quad \beta=0,1,2,3,4,
$$

that is,

$$
\begin{aligned}
& \rho_{t}+\operatorname{div}_{x}(\rho u)=0, \\
& \left(\rho u_{i}\right)_{t}+\sum_{j} \partial_{x_{j}}\left(\rho u_{i} u_{j}\right)+\partial_{x_{i}}(\rho T)+\partial_{x_{j}} \int_{\mathbb{R}^{3}} G v_{i} v_{j} \mathrm{~d} v=0, \quad i=1,2,3, \\
& {\left[\rho\left(\frac{|u|^{2}}{2}+\frac{3 T}{2}\right)\right]_{t}+\sum_{j} \partial_{x_{j}}\left[u_{j}\left(\frac{5}{2} \rho T+\frac{1}{2} \rho|u|^{2}\right)\right]+\int_{\mathbb{R}^{3}} \frac{1}{2}|v|^{2} v \cdot \nabla_{x} G \mathrm{~d} v=0 .}
\end{aligned}
$$

This is a fluid-type system, coupled with the microscopic part $G$, which satis- 
fies the microscopic equation derived by applying the microscopic projection $P_{1}^{M}$ to Equation (1.1):

$$
\begin{aligned}
& G_{t}+P_{1}^{M}\left(v \cdot \nabla_{x} M+v \cdot \nabla_{x} G\right) \\
& =\rho^{\alpha} \nabla_{v} \cdot\left(T \nabla_{v} M+(v-u) M\right)+\rho^{\alpha} \nabla_{v} \cdot\left(T \nabla_{v} G+(v-u) G\right) \\
& =\rho^{\alpha} \nabla_{v} \cdot\left(T \nabla_{v} G+(v-u) G\right) .
\end{aligned}
$$

Note that

$$
\nabla_{v}\left(\frac{g}{M}\right)=\frac{\nabla_{v} g}{M}-\frac{(v-u) g}{T M}, \nabla_{v} \cdot\left(T \nabla_{v} g+(v-u) g\right)=T \nabla_{v}\left[M \cdot \nabla_{v}\left(\frac{g}{M}\right)\right],
$$

then (3.3) can be rewritten as

$$
G_{t}+P_{1}^{M}\left(v \cdot \nabla_{x} M+v \cdot \nabla_{x} G\right)=: \rho^{\alpha} L_{M} G,
$$

where $L_{M}$ is the Fokker-Planck operator given by

$$
L_{M} g:=T \nabla_{v}\left[M \cdot \nabla_{v}\left(\frac{g}{M}\right)\right] \text {. }
$$

In summary, the nonlinear Fokker-Planck Equation (1.1) is decomposed into the fluid-type system (3.2) coupled with the microscopic Equation (3.4). Note that the system (3.2) becomes Euler equations when the microscopic part $G$ is set to be zero, as in the traditional Hilbert expansion. To recover Navier-Stokes type system [25] [26], one need to analyze more carefully the viscosity and heat diffusion from the microscope part, similar to the Chapman-Enskog expansion for Boltzmann equation. The details of the computation will be given in next section.

\section{Derivation of the Viscosity and Heat Diffusion}

Note that the nonlocal integration terms in the fluid-type system (3.2) are related to the microscopic component $G$, which are the origin of viscosity and heat diffusion. This is derived by applying Chapman-Enskog expansion as for Boltzmann equation, keeping the leading order term in the microscopic part (see [25] [26]). Before analyzing the microscopic component $G$, we give the following basic properties of the Fokker-Planck operator.

Lemma 4.1. The Fokker-Planck operator $L_{M}$ defined in (3.5) satisfies

$$
\begin{aligned}
& L_{M} \chi_{0}^{M}=0, L_{M} \chi_{i}^{M}=-\chi_{i}^{M}(i=1,2,3), L_{M} \chi_{4}^{M}=-2 \chi_{4}^{M}, \\
& \left\langle L_{M} g, h\right\rangle_{M}=\left\langle g, L_{M} h\right\rangle_{M}, \\
& -\left\langle P_{1}^{M} h, L_{M} P_{1}^{M} h\right\rangle_{M} \geq \lambda_{0}\left\|P_{1}^{M} h\right\|_{M, v}^{2} \text { for some constant } \lambda_{0}>0, \\
& \left\|L_{M}^{-1} P_{1}^{M} h\right\|_{M, v}^{2} \leq \frac{1}{\lambda_{0}^{2}} \iint_{\mathbb{R}^{3} \times \mathbb{R}^{3}}\left|P_{1}^{M} h\right|^{2} \frac{\left(1+|v|^{2}\right)^{-1}}{M} \mathrm{~d} v \mathrm{~d} x,
\end{aligned}
$$

and

$$
L_{M} N_{1}=-2 N_{1}, L_{M} N_{2}=-3 N_{2} \text {, }
$$

where 


$$
\begin{aligned}
& N_{1}:=\sum_{i j}\left(v_{i}-u_{i}\right)\left(v_{j}-u_{j}\right) u_{x_{j}}^{i} \frac{M}{T}-\frac{\nabla_{x} \cdot u}{3 T}|v-u|^{2} M, \\
& N_{2}:=\left(|v-u|^{2}-5 T\right)\left(v_{i}-u_{i}\right) M .
\end{aligned}
$$

Proof. The proof of (4.1) is direct. Note that this is also similar to the microscopic version of the $\mathrm{H}$-theorem for Boltzmann equation in [25]. Next, we compute

$$
\begin{aligned}
& L_{M}\left[\left(v_{i}-u_{i}\right)\left(v_{j}-u_{j}\right) M\right] \\
& =T \nabla_{v} \cdot\left[M \nabla_{v}\left(\left(v_{i}-u_{i}\right)\left(v_{j}-u_{j}\right)\right)\right] \\
& =\sum_{k} T \partial_{v_{k}}\left[M \delta_{k j}\left(v_{i}-u_{i}\right)+M \delta_{k j}\left(v_{j}-u_{j}\right)\right] \\
& =\left[-\left(v_{i}-u_{i}\right)\left(v_{j}-u_{j}\right) M+\sum_{k} M T \delta_{k j} \delta_{k i}\right] * 2,
\end{aligned}
$$

that is, for fixed $i, j$,

$$
\begin{aligned}
& L_{M}\left[\left(v_{i}-u_{i}\right)\left(v_{j}-u_{j}\right) M\right] \\
& =-2\left(v_{i}-u_{i}\right)\left(v_{j}-u_{j}\right) M+2 \delta_{i j} T M .
\end{aligned}
$$

Next,

$$
\begin{aligned}
L_{M}\left(|v-u|^{2} M\right) & =T \nabla_{v} \cdot\left[M \nabla_{v} \cdot\left(\frac{|v-u|^{2}}{M} M\right)\right] \\
& =2 T \nabla_{v} \cdot[(v-u) M] \\
& =-2|v-u|^{2} M+6 T M .
\end{aligned}
$$

Combining (4.3) and (4.4) to get

$$
\begin{aligned}
& L_{M}\left[\sum_{i j}\left(v_{i}-u_{i}\right)\left(v_{j}-u_{j}\right) u_{x_{j}}^{i} \frac{M}{T}-\frac{\nabla_{x} \cdot u}{3 T}|v-u|^{2} M\right] \\
& =\sum_{i j}\left[-2\left(v_{i}-u_{i}\right)\left(v_{j}-u_{j}\right) u_{x_{j}}^{i} \frac{M}{T}+2 \delta_{i j} u_{x_{j}}^{i} \frac{T M}{T}\right]-\frac{\nabla_{x} \cdot u}{3 T}\left(-2|v-u|^{2} M+6 T M\right) \\
& =-2\left[\sum_{i j}\left(v_{i}-u_{i}\right)\left(v_{j}-u_{j}\right) u_{x_{j}}^{i} \frac{M}{T}-\frac{\nabla_{x} \cdot u}{3 T}|v-u|^{2} M\right],
\end{aligned}
$$

thus the first equation in (4.2) is proved. Next,

$$
\begin{aligned}
L_{M}\left(\left(v_{i}-u_{i}\right) M\right) & =T \nabla_{v}\left[M \nabla_{v}\left(v_{i}-u_{i}\right)\right] \\
& =\sum_{j} T \partial_{v_{j}}\left[M \partial_{v_{j}}\left(v_{i}-u_{i}\right)\right] \\
& =T \partial_{v_{i}} M \\
& =-\left(v_{i}-u_{i}\right) M,
\end{aligned}
$$

then

$$
L_{M}\left[5 T\left(v_{i}-u_{i}\right) M\right]=-5\left(v_{i}-u_{i}\right) T M .
$$

Furthermore, 


$$
\begin{aligned}
& L_{M}\left(\left(v_{i}-u_{i}\right)|v-u|^{2} M\right)=T \nabla_{v} \cdot\left[M \nabla_{v}\left(\left(v_{i}-u_{i}\right)|v-u|^{2}\right)\right] \\
&=T \partial_{v_{j}}\left[M \delta_{i j}|v-u|^{2}+2 M\left(v_{i}-u_{i}\right)\left(v_{j}-u_{j}\right)\right] \\
&=T \partial_{v_{j}}\left(M|v-u|^{2}\right)+2 T \partial_{v_{j}}\left(M\left(v_{i}-u_{i}\right)\left(v_{j}-u_{j}\right)\right) \\
&=-\left(v_{i}-u_{i}\right)|v-u|^{2} M+2\left(v_{i}-u_{i}\right) T M-2|v-u|^{2}\left(v_{i}-u_{i}\right) M \\
&+6 T\left(v_{i}-u_{i}\right) M+2 T M\left(v_{i}-u_{i}\right) \\
&=-3\left(v_{i}-u_{i}\right)|v-u|^{2} M+10\left(v_{i}-u_{i}\right) T M .
\end{aligned}
$$

Combine (4.7)-(4.8):

$$
L_{M}\left[\left(|v-u|^{2}-5 T\right)\left(v_{i}-u_{i}\right) M\right]=-3\left(|v-u|^{2}-5 T\right)\left(v_{i}-u_{i}\right) M,
$$

this is the second equation in (4.2).

Now, using the properties of the Fokker-Planck operator $L_{M}$, one can invert $L_{M}$ on (3.4) to get

$$
G=\rho^{-\alpha} L_{M}^{-1}\left(P_{1}^{M}\left(v \cdot \nabla_{x} M\right)\right)+\rho^{-\alpha} L_{M}^{-1} \pi, \pi:=G_{t}+P_{1}^{M}\left(v \cdot \nabla_{\chi} G\right) .
$$

By substituting (4.10) into (3.2), one has the fluid-type equations

$$
\begin{aligned}
& \rho_{t}+\operatorname{div}_{x}(\rho u)=0, \\
& \left(\rho u_{i}\right)_{t}+\sum_{j} \partial_{x_{j}}\left(\rho u_{i} u_{j}\right)+\partial_{x_{i}}(\rho T) \\
& =-\sum_{j} \partial_{x_{j}} \int_{\mathbb{R}^{3}} \rho^{-\alpha} L_{M}^{-1} \pi v_{i} v_{j} \mathrm{~d} v-\sum_{j} \partial_{x_{j}} \int_{\mathbb{R}^{3}} \rho^{-\alpha} L_{M}^{-1}\left(P_{1}^{M}\left(v \cdot \nabla_{x} M\right)\right) v_{i} v_{j} \mathrm{~d} v, \\
& {\left[\rho\left(\frac{|u|^{2}}{2}+\frac{3 T}{2}\right)\right]_{t}+\sum_{j} \partial_{x_{j}}\left[u_{j}\left(\frac{5}{2} \rho T+\frac{1}{2} \rho|u|^{2}\right)\right]} \\
& =-\sum_{i} \partial_{x_{j}} \int_{\mathbb{R}^{3}} \frac{1}{2}|v|^{2} v_{i} \rho^{-\alpha} L_{M}^{-1} \pi \mathrm{d} v-\sum_{i} \partial_{x_{i}} \int_{\mathbb{R}^{3}} \frac{1}{2}|v|^{2} v_{i} \rho^{-\alpha} L_{M}^{-1}\left(P_{1}^{M}\left(v \cdot \nabla_{x} M\right)\right) \mathrm{d} v .
\end{aligned}
$$

As the same to the Boltzmann equation, the terms containing $L_{M}^{-1}\left(P_{1}^{M}\left(v \cdot \nabla_{x} M\right)\right)$ in above yield the viscosity and heat conductivity, which can be specified more precisely, by using the following lemma.

Lemma 4.2. The Fokker-Planck operator $L_{M}$ defined in (3.5) satisfies

$$
\begin{aligned}
L_{M}^{-1} P_{1}^{M}\left(v \cdot \nabla_{x} M\right)= & -\frac{1}{6 T^{2}}\left(|v-u|^{2}-5 T\right)(v-u) \cdot \nabla T M \\
& -\frac{1}{2 T}\left[\sum_{i j}\left(v_{i}-u_{i}\right)\left(v_{j}-u_{j}\right) u_{x_{j}}^{i}-\frac{\nabla \cdot u}{3}|v-u|^{2} M\right] .
\end{aligned}
$$

Proof. Notice that the $x$-derivative of the local Maxwellian $M$ in (2.2) is

$$
\begin{aligned}
\partial_{x_{i}} M & =\left[\frac{\rho_{x_{i}}}{\rho}-\frac{3}{2} \frac{T_{x_{i}}}{T}-\left(\frac{|v-u|^{2}}{2 T}\right)_{x_{i}}\right] M \\
& =\left[\frac{\rho_{x_{i}}}{\rho}-\frac{3}{2} \frac{T_{x_{i}}}{T}+\frac{T_{x_{i}}}{2 T^{2}}|v-u|^{2}+\frac{(v-u) u_{x_{i}}}{T}\right] M,
\end{aligned}
$$

then 


$$
\begin{aligned}
P_{1}^{M}\left(v \cdot \nabla_{x} M\right)= & P_{1}^{M}\left((v-u) \cdot \nabla_{x} M\right)+P_{1}^{M}\left(u \cdot \nabla_{x} M\right) \\
= & P_{1}^{M}\left(\left(v_{i}-u_{i}\right) \frac{T_{x_{i}}}{2 T^{2}}|v-u|^{2} M\right) \\
& +P_{1}^{M}\left(\left(v_{i}-u_{i}\right)\left(v_{j}-u_{j}\right) u_{x_{j}}^{i} \frac{M}{T}\right) \\
:= & I_{1}+I_{2} .
\end{aligned}
$$

Use the definition of the microscopic projection, one has

$$
\begin{aligned}
I_{1} & =(v-u) \cdot \nabla T \frac{|v-u|^{2}}{2 T^{2}} M-\int_{\mathbb{R}^{3}}\left(v_{j}-u_{j}\right)\left(v_{i}-u_{i}\right) \frac{T_{x_{i}}}{2 T^{2}}|v-u|^{2} M \mathrm{~d} v \cdot \frac{v_{j}-u_{j}}{T \rho} M \\
& =\left(\frac{|v-u|^{2}}{2 T^{2}}-\frac{5}{2 T}\right)(v-u) \cdot \nabla T M .
\end{aligned}
$$

and

$$
\begin{aligned}
I_{2}= & \left(v_{i}-u_{i}\right)\left(v_{j}-u_{j}\right) u_{x_{j}}^{i} \frac{M}{T}-\left(\int_{\mathbb{R}^{3}}\left(v_{i}-u_{i}\right)\left(v_{j}-u_{j}\right) u_{x_{j}}^{i} \frac{M}{T} \mathrm{~d} v\right) \frac{M}{\rho} \\
& -\left(\int_{\mathbb{R}^{3}}\left(v_{i}-u_{i}\right)\left(v_{j}-u_{j}\right) u_{x_{j}}^{i} \frac{M}{T} \cdot\left(\frac{|v-u|^{2}}{T}-3\right) \mathrm{d} v\right) \frac{1}{6 \rho}\left(\frac{|v-u|^{2}}{T}-3\right) M \\
: & I_{20}+I_{21}+I_{22} .
\end{aligned}
$$

Note that

$$
\int_{\mathbb{R}^{3}}\left|v_{i}-u_{i}\right|^{2} M \mathrm{~d} v=\frac{1}{3} \int_{\mathbb{R}^{3}}|v-u|^{2} M \mathrm{~d} v=\rho T, \int_{\mathbb{R}^{3}}\left|v_{i}-u_{i}\right|^{4} M \mathrm{~d} v=15 \rho T^{2},
$$

then

$$
I_{21}=\sum_{i} \int_{\mathbb{R}^{3}}\left|v_{i}-u_{i}\right|^{2} M \mathrm{~d} v \cdot \frac{1}{T} u_{x_{i}}^{i} \cdot \frac{M}{\rho}=(\nabla \cdot u) M,
$$

and

$$
\begin{aligned}
I_{22} & =\sum_{i}\left(\int_{\mathbb{R}^{3}}\left|v_{i}-u_{i}\right|^{2}\left(\frac{|v-u|^{2}}{T}-3\right) M \mathrm{~d} v\right) \frac{u_{x_{i}}^{i}}{6 \rho T}\left(\frac{|v-u|^{2}}{T}-3\right) M \\
& =\frac{1}{3}\left(\int_{\mathbb{R}^{3}}|v-u|^{2}\left(\frac{|v-u|^{2}}{T}-3\right) M \mathrm{~d} v\right) \frac{\nabla \cdot u}{6 \rho T}\left(\frac{|v-u|^{2}}{T}-3\right) M \\
& =\frac{\nabla \cdot u}{3}\left(\frac{|v-u|^{2}}{T}-3\right) M .
\end{aligned}
$$

Combining the above to get

$$
I_{2}=\sum_{i j}\left(v_{i}-u_{i}\right)\left(v_{j}-u_{j}\right) u_{x_{j}}^{i} \frac{M}{T}-\frac{\nabla \cdot u}{3 T}|v-u|^{2} M .
$$

Plug (4.14) and (4.18) into (4.13), one gets

$$
\begin{aligned}
P_{1}^{M}\left(v \cdot \nabla_{x} M\right)= & \left(\frac{|v-u|^{2}}{2 T^{2}}-\frac{5}{2 T}\right)(v-u) \cdot \nabla T M \\
& +\sum_{i j}\left(v_{i}-u_{i}\right)\left(v_{j}-u_{j}\right) u_{x_{j}}^{i} \frac{M}{T}-\frac{\nabla \cdot u}{3 T}|v-u|^{2} M .
\end{aligned}
$$


Then (4.12) is proved with the help of Lemma 4.1.

Now we compute the viscosity and heat conductivity, which are given in below.

Theorem 4.3. For each index $i, j$, we have

$$
\begin{gathered}
\int_{\mathbb{R}^{3}} L_{M}^{-1} P_{1}^{M}\left(v \cdot \nabla_{x} M\right) v_{i} v_{j} \mathrm{~d} v=-\frac{\rho T}{2}\left(u_{x_{j}}^{i}+u_{x_{i}}^{j}-\frac{2}{3}(\operatorname{div} u) \delta_{i j}\right) . \\
\int_{\mathbb{R}^{3}} L_{M}^{-1} P_{1}^{M}\left(v \cdot \nabla_{x} M\right) v_{j}|v|^{2} \mathrm{~d} v \\
=-\frac{5}{3} \rho T \partial_{x_{j}} T-\rho T \sum_{i} u_{i}\left(u_{x_{j}}^{i}+u_{x_{i}}^{j}-\frac{2}{3}(\nabla \cdot u) \delta_{i j}\right) .
\end{gathered}
$$

Remark: The right hand sides of (4.20) and (4.21), respectively, give the viscosity and heat conductivity to the fluid-type system.

Proof. Using (4.12), one compute

$$
\begin{aligned}
& \int_{\mathbb{R}^{3}} L_{M}^{-1} P_{1}^{M}\left(v \cdot \nabla_{x} M\right) v_{i} v_{j} \mathrm{~d} v \\
& =-\frac{1}{6 T^{2}} \int_{\mathbb{R}^{3}}\left(|v-u|^{2}-5 T\right)(v-u) \cdot \nabla T v_{i} v_{j} M \mathrm{~d} v \\
& \quad-\int_{\mathbb{R}^{3}} \frac{1}{2 T}\left[\sum_{k l}\left(v_{k}-u_{k}\right)\left(v_{l}-u_{l}\right) u_{x_{k}}^{l}-\frac{\nabla \cdot u}{3}|v-u|^{2} v_{i} v_{j} M \mathrm{~d} v\right] \\
& :=J_{1}+J_{2},
\end{aligned}
$$

where

$$
\begin{aligned}
J_{1}= & -\frac{1}{6 T^{2}} \int_{\mathbb{R}^{3}}\left(|v-u|^{2}-5 T\right)(v-u) \cdot \nabla T\left(v_{i}-u_{i}\right)\left(v_{j}-u_{j}\right) M \mathrm{~d} v \\
& -\frac{1}{6 T^{2}} \int_{\mathbb{R}^{3}}\left(|v-u|^{2}-5 T\right)(v-u) \cdot \nabla T\left(u_{i}\left(v_{j}-u_{j}\right)+\left(v_{i}-u_{i}\right) u_{j}+u_{i} u_{j}\right) M \mathrm{~d} v \\
= & -\frac{u_{i}}{6 T^{2}} \int_{\mathbb{R}^{3}}\left(|v-u|^{2}-5 T\right)(v-u) \cdot \nabla T\left(v_{j}-u_{j}\right) M \mathrm{~d} v-\cdots(i \leftrightarrow j) \\
= & -\frac{u_{i} \partial_{x_{j}} T}{6 T^{2}} \int_{\mathbb{R}^{3}}\left(|v-u|^{2}-5 T\right)\left|v_{j}-u_{j}\right|^{2} M \mathrm{~d} v-\cdots \\
= & -\frac{u_{i} \partial_{\chi_{j}} T}{18 T^{2}} \int_{\mathbb{R}^{3}}\left(|v-u|^{4}-5 T|v-u|^{2}\right) M \mathrm{~d} v \\
= & 0,
\end{aligned}
$$

and

$$
\begin{aligned}
J_{2}= & -\int_{\mathbb{R}^{3}} \frac{1}{2 T}\left[\sum_{k l}\left(v_{k}-u_{k}\right)\left(v_{l}-u_{l}\right) u_{x_{k}}^{l}-\frac{\nabla \cdot u}{3}|v-u|^{2}\right]\left(v_{i}-u_{i}\right)\left(v_{j}-u_{j}\right) M \mathrm{~d} v \\
& -\int_{\mathbb{R}^{3}} \frac{1}{2 T}\left[\sum_{k l}\left(v_{k}-u_{k}\right)\left(v_{l}-u_{l}\right) u_{x_{k}}^{l}-\frac{\nabla \cdot u}{3}|v-u|^{2}\right] \\
& \times\left(u_{i}\left(v_{j}-u_{j}\right)+\left(v_{i}-u_{j}\right) u_{i}+u_{i} u_{i}\right) M \mathrm{~d} v \\
= & -\int_{\mathbb{R}^{3}} \frac{1}{2 T}\left[\sum_{k l}\left(v_{k}-u_{k}\right)\left(v_{l}-u_{l}\right) u_{x_{k}}^{l}-\frac{\nabla \cdot u}{3}|v-u|^{2}\right]\left(\left|v_{i}-u_{i}\right|^{2} \delta_{i j}+u_{i} u_{j}\right) M \mathrm{~d} v \\
= & -\frac{\rho T}{2}\left(u_{x_{j}}^{i}+u_{x_{i}}^{j}-\frac{2}{3}(\operatorname{divu}) \delta_{i j}\right) .
\end{aligned}
$$

Combining (4.22)-(4.24), one has (4.20). Next, for fixed index $j$, 


$$
\begin{aligned}
& \int_{\mathbb{R}^{3}} L_{M}^{-1} P_{1}^{M}\left(v \cdot \nabla_{x} M\right) v_{j}|v|^{2} \mathrm{~d} v \\
& =-\frac{\partial_{x_{i}} T}{6 T^{2}} \int_{\mathbb{R}^{3}}\left(|v-u|^{2}-5 T\right)\left(v_{i}-u_{i}\right) v_{j}|v|^{2} M \mathrm{~d} v \\
& -\int_{\mathbb{R}^{3}} \frac{1}{2 T}\left[\sum_{k l}\left(v_{k}-u_{k}\right)\left(v_{l}-u_{l}\right) u_{x_{k}}^{l}-\frac{\nabla \cdot u}{3}|v-u|^{2}\right] v_{j}|v|^{2} M \mathrm{~d} v \\
& :=J_{3}+J_{4},
\end{aligned}
$$

in which

$$
\begin{aligned}
J_{3}= & -\frac{\partial_{x_{i}} T}{6 T^{2}} \int_{\mathbb{R}^{3}}\left(|v-u|^{2}-5 T\right)\left(v_{i}-u_{i}\right)\left(v_{j}-u_{j}\right)|v|^{2} M \mathrm{~d} v \\
& -\frac{u_{j} \partial_{x_{i}} T}{6 T^{2}} \int_{\mathbb{R}^{3}}\left(|v-u|^{2}-5 T\right)\left(v_{i}-u_{i}\right)|v|^{2} M \mathrm{~d} v \\
= & -\frac{5}{3} \rho T \partial_{x_{j}} T
\end{aligned}
$$

and

$$
\begin{aligned}
J_{4}= & -\frac{1}{2 T} \int_{\mathbb{R}^{3}}\left[\sum_{k l}\left(v_{k}-u_{k}\right)\left(v_{l}-u_{l}\right) u_{x_{k}}^{l}-\frac{\nabla \cdot u}{3}|v-u|^{2}\right]\left(v_{j}-u_{j}\right)|v|^{2} M \mathrm{~d} v \\
& -\frac{u_{j}}{2 T} \int_{\mathbb{R}^{3}}\left[\sum_{k l}\left(v_{k}-u_{k}\right)\left(v_{l}-u_{l}\right) u_{x_{k}}^{l}-\frac{\nabla \cdot u}{3}|v-u|^{2}\right]|v|^{2} M \mathrm{~d} v \\
= & -\rho T \sum_{i} u_{i}\left(u_{x_{j}}^{i}+u_{x_{i}}^{j}-\frac{2}{3}(\nabla \cdot u) \delta_{i j}\right) .
\end{aligned}
$$

Put (4.26) and (4.27) into (4.25), one gets (4.21).

Now with the help of Theorem 4.3, the macroscopic Equation (4.11) becomes

$$
\begin{aligned}
& \rho_{t}+\operatorname{div}_{x}(\rho u)=0 \\
& \left(\rho u_{i}\right)_{t}+\sum_{j} \partial_{x_{j}}\left(\rho u_{i} u_{j}\right)+\partial_{x_{i}}(\rho T) \\
& =\sum_{j} \partial_{x_{j}}\left(\frac{\rho^{1-\beta} T}{2}\left(u_{x_{j}}^{i}+u_{x_{i}}^{j}-\frac{2}{3}(\operatorname{div} u) \delta_{i j}\right)\right)-\sum_{j} \partial_{x_{j}} \int_{\mathbb{R}^{3}} \rho^{-\alpha} L_{M}^{-1} \pi v_{i} v_{j} \mathrm{~d} v, \\
& {\left[\rho\left(\frac{|u|^{2}}{2}+\frac{3 T}{2}\right)\right]_{t}+\nabla \cdot\left[u\left(\frac{5}{2} \rho T+\frac{1}{2} \rho|u|^{2}\right)\right]} \\
& =-\sum_{i} \partial_{x_{i}} \int_{\mathbb{R}^{3}} \frac{1}{2}|v|^{2} v_{i} \rho^{-\alpha} L_{M}^{-1} \pi \mathrm{d} v+\frac{5}{12} \nabla \cdot\left[\rho^{1-\beta} \nabla\left(T^{2}\right)\right] \\
& \quad+\sum_{i, j} \partial_{x_{j}}\left(\frac{\rho^{1-\beta} T}{2} u_{i}\left(u_{x_{j}}^{i}+u_{x_{i}}^{j}-\frac{2}{3}(\operatorname{div} u) \delta_{i j}\right)\right) .
\end{aligned}
$$

Note that this is a Navier-Stokes type fluid system, coupled with $\pi$ defined in (4.10), the remainder term of the microscopic component.

\section{Conclusion}

The fully non-linear Fokker-Planck Equation (1.1) preserves mass, momentum and energy; the dissipation is much weaker than that in the simplified model considered in [10]. By decomposing the unknown function around the local Maxwellian, the Fokker-Planck equation is rewritten into a fluid-type system 
(4.28), which includes viscosity and heat diffusion giving dissipative mechanism of the system. The research tools for fluid-type system can be applied to further study of the fully non-linear Fokker-Planck equation, for instance, the stability of global equilibrium or the wave patterns to the Fokker-Planck equation, which will be the pursuit of our future study.

\section{Acknowledgements}

This research is partially supported by National Natural Science Foundation of China (Nos. 11871335 and 11971008) and USRP of East China University of Science and Technology (No. X20251).

\section{Conflicts of Interest}

The authors declare no conflicts of interest regarding the publication of this paper.

\section{References}

[1] Bellomo, N., Knopoff, D. and Soler, J. (2013) On the Difficult Interplay between Life, Complexity and Mathematical Sciences. Mathematical Models and Methods in Applied Sciences, 23, 1861-1913. https://doi.org/10.1142/S021820251350053X

[2] Bellouquid, A., De Angelis, E. and Knopoff, D. (2013) From the Modelling of the Immune Hallmarks of Cancer to a Black Swan in Biology. Mathematical Models and Methods in Applied Sciences, 23, 949-978.

https://doi.org/10.1142/S0218202512500650

[3] Carrillo, J.A. and Toscani, G. (1998) Exponential Convergence toward Equilibrium for Homogeneous Fokker-Planck-Type Equations. Mathematical Methods in the Applied Sciences, 21, 1269-1286. https://doi.org/10.1002/(SICI)1099-1476(19980910)21:13<1269::AID-MMA995>3.0. CO;2-O

[4] Frank, T.D. (2005) Nonlinear Fokker-Planck Equations: Fundamentals and Applications. Springer, Berlin.

[5] Furioli, G., Pulvirenti, A., Terraneo, E. and Toscani, G. (2017) Fokker-Planck Equations in the Modelling of Socio-Economic Phenomena. Mathematical Models and Methods in Applied Sciences, 27, 115-158.

https://doi.org/10.1142/S0218202517400048

[6] Naldi, G., Pareschi, L. and Toscani, G. (2010) Mathematical Modeling of Collective Behavior in Socio-Economic and Life Sciences. Springer Verlag, Heidelberg. https://doi.org/10.1007/978-0-8176-4946-3

[7] Villani, C. (2002) A Review of Mathematical Topics in Collisional Kinetic Theory. In: Handbook of Mathematical Fluid Dynamics, Vol. I, North-Holland, Amsterdam, 71-305. https://doi.org/10.1016/S1874-5792(02)80004-0

[8] Galkin, V.S. and Rusakov, S.V. (2007) Kinetic Fokker-Planck Equation for FreeMolecular, Thermally Nonequilibrium Brownian Particles in an Inhomogeneous Gas. Fluid Dynamics, 42, 330-334. https://doi.org/10.1134/S0015462807020184

[9] Montgomery, D. (1971) Brownian Motion from Boltzmann's Equation. The Physics of Fluids, 14, 2088-2090. https://doi.org/10.1063/1.1693297 
[10] Liao, J., Wang, Q.-R. and Yang, X.-F. (2018) Global Existence and Decay Rates of the Solutions near Maxwellian for Non-Linear Fokker-Planck Equations. Journal of Statistical Physics, 173, 222-241. https://doi.org/10.1007/s10955-018-2129-3

[11] Chacón-Acosta, G. and Kremer, G.M. (2007) Fokker-Planck-Type Equations for a Simple Gas and for a Semirelativistic Brownian Motion from a Relativistic Kinetic Theory. Physical Review E, 76, Article ID: 021201. https://doi.org/10.1103/PhysRevE.76.021201

[12] Félix, J.A.A. and Calogero, S. (2017) On a Relativistic Fokker-Planck Equation in Kinetic Theory. Kinetic and Related Models, 4, 401-426. https://doi.org/10.3934/krm.2011.4.401

[13] Luo, L. and Zhang, X. (2015) Global Classical Solutions for Quantum Kinetic Fokker-Planck Equations. Acta Mathematica Scientia, 35B, 140-156. https://doi.org/10.1016/S0252-9602(14)60147-8

[14] Yano, R. (2015) On Quantum Fokker-Planck Equation. Journal of Statistical Physics, 158, 1-17. https://doi.org/10.1007/s10955-014-1123-7

[15] DiPerna, R.J. and Lions, P.-L. (1988) On the Fokker-Planck-Boltzmann Equation. Communications in Mathematical Physics, 120, 1-23.

https://doi.org/10.1007/BF01223204

[16] Li, H. and Matsumura, A. (2008) Behaviour of the Fokker-Planck-Boltzmann Equation near a Maxwellian. Archive for Rational Mechanics and Analysis, 189, 1-44. https://doi.org/10.1007/s00205-007-0057-5

[17] Zhong, M. and Li, H. (2014) Long Time Behavior of the Fokker-Planck-Boltzmann Equation. Acta Mathematicae Applicatae Sinica, 30, 533-554. https://doi.org/10.1007/s10255-012-0158-2

[18] Perthame, B. (1990) Higher Moments for Kinetic Equations: The Vlasov-Poisson and Fokker-Planck Cases. Mathematical Methods in the Applied Sciences, 13, 441452. https://doi.org/10.1002/mma.1670130508

[19] Hwang, H.J. and Jang, J. (2013) On the Vlasov-Poisson-Fokker-Planck Equation near Maxwellian. Discrete and Continuous Dynamical Systems Series B, 18, 681-691. https://doi.org/10.3934/dcdsb.2013.18.681

[20] Yang, T. and Yu, H. (2010) Global Classical Solutions for the Vlasov-MaxwellFokker-Planck System. SIAM Journal on Mathematical Analysis, 42, 459-488. https://doi.org/10.1137/090755796

[21] Carrillo, J.A., Duan, R. and Moussa, A. (2011) Global Classical Solutions Close to Equilibrium the Vlasov-Fokker-Planck-Euler System. Kinetic and Related Models, 4, 227-258. https://doi.org/10.3934/krm.2011.4.227

[22] Goudon, T., He, L., Moussa, A. and Zhang, P. (2009) The Navier-Stokes-VlasovFokker-Planck System near Equilibrium. SIAM Journal on Mathematical Analysis, 42, 2177-2202. https://doi.org/10.1137/090776755

[23] Li, F., Mu, Y. and Wang, D. (2017) Strong Solutions to the Compressible Navier-Stokes-Vlasov-Fokker-Planck Equations: Global Existence near the Equilibrium and Large Time Behavior. SIAM Journal on Mathematical Analysis, 49, 984-1026. https://doi.org/10.1137/15M1053049

[24] Mellet, A. and Vasseur, A. (2008) Asymptotic Analysis for a Vlasov-FokkerPlanck/Compressible Navier-Stokes System of Equations. Communications in Mathematical Physics, 281, 573-596. https://doi.org/10.1007/s00220-008-0523-4

[25] Liu, T.-P., Yang, T. and Yu, S.-H. (2004) Energy Method for the Boltzmann Equa- 
tion. Physica D, 188, 178-192. https://doi.org/10.1016/j.physd.2003.07.011

[26] Chapman, S. and Cowling, T. (1990) The Mathematical Theory of Non-Uniform Gases. 3rd Edition, Cambridge University Press, Cambridge.

\section{Nomenclatures}

the distribution of particles at time $t$, position $x$ and velocity $v$. $f(t, x, v)$, collision invariants: $\psi_{0}=1, \psi_{i}=v_{i}(i=1,2,3), \psi_{4}=\frac{1}{2}|v|^{2}$, mass density, the mean velocity and the local temperature: $(\rho, \tilde{u}, T)$, perturbation of mass density, the mean velocity and the local temperature: $(\sigma, u, \tilde{T})$, local/global Maxwellian: $M(t, x, v), \bar{M}(t, x, v)$, weighted inner product with respect to a Maxwellian $M$ :

$$
\langle g, h\rangle_{M}:=\int_{\mathbb{R}^{3}} g(v) h(v) \frac{1}{M} \mathrm{~d} v,
$$

basis function of the macroscopic quantities:

$$
\chi_{0}^{M}=\frac{1}{\sqrt{\rho}} M, \chi_{i}^{M}=\frac{v_{i}-u_{i}}{\sqrt{T \rho}} M(i=1,2,3), \quad \chi_{4}^{M}=\frac{1}{\sqrt{6 \rho}}\left(\frac{|v-u|^{2}}{T}-3\right) M,
$$

macroscopic projection $P_{0}^{M}$ and microscopic projection $P_{1}^{M}$ of a function $h$ :

$$
P_{0}^{M} h:=\sum_{\beta=0}^{4}\left\langle h, \chi_{\beta}^{M}\right\rangle_{M} \chi_{\beta}^{M}, P_{1}^{M} h=\left(I-P_{0}^{M}\right) h,
$$

macro-micro decomposition of $f . \quad f(t, x, v)=M(t, x, v)+G(t, x, v)$,

Fokker-Planck operator: $L_{M} g:=T \nabla_{v}\left[M \cdot \nabla_{v}\left(\frac{g}{M}\right)\right]$. 